\title{
El gandul (Cajanus cajan (L.) Mill sp.) una excelente alternativa para Sistemas Agrosilvopastoriles
}

\author{
Luis Alonso González, Alfonso Rey \& Aarón Fallas \\ Universidad Estatal a Distancia, Escuela de Ciencias Exactas y Naturales; luarce@uned.ac.cr; arey@uned.ac.cr; \\ afallas@uned.ac.cr
}

Recibido: 19 abril 2016

\section{RESUMEN}

El gandul (Cajanus cajan (l.) Millsp.) es una leguminosa (Fabaceae) que puede ser parte de los modelos productivos de fincas integrales bajo el esquema de sistemas agrosilvopastoriles, que además de proporcionar una opción para mejorar la dieta familiar incorporando un producto nuevo y rico en nutrientes, proporcionan también una oportunidad para generar ingresos extras al núcleo familiar, además de contribuir con la seguridad alimentaria y nutricional, así como a la recuperación y protección del recurso hídrico. El arbusto de gandul es muy resistente a las inclemencias del tiempo y una especie con muchas variedades, de las cuales en Costa Rica se han trabajado al menos seis. Se utiliza para descompactar el suelo, para alimentación humana y animal, agente de control biológico y posee un alto valor nutritivo. Se concluye que esta leguminosa es de gran potencial para uso en suelos, en cuencas hidrográficas y en alimentación humana o animal. El proyecto busca incorporar en la economía y la dieta familiar, en la zona de Peñas Blancas, San Carlos, un producto que mejora no solo las condiciones actuales de los grupos familiares productores de cultivos, sino además una especie que contribuya a mejorar las condiciones ambientales. Este objetivo se ha logrado alcanzar con la aclimatación en la zona de al menos 6 variedades de Gandul, con resultados importantes en lo concerniente a producción y mejoramiento de suelos.

Palabras clave: Fijación de Nitrógeno, Dieta Familiar, Sistemas Agrosilvopastoriles, Seguridad Alimentaría y Nutricional, protección de suelos.

\footnotetext{
ABSTRACT

Pigeon pea (Cajanus cajan (1.) Mill sp.) is a legume that can be part of the productive models of integral farms under an agrosilvopastoryl system, which resides being an option to improve the diet since it has a high nutricional value and it's new, also represents an opportunity to generate extra income to familias that own a small company or farm. It also contributes to food security as well as the protection of water basins. Due to its resistance to wheter, it's an option
}

Aceptado: 13 junio 2016

to substitute legumes such as beans, lentils or chickpeas. There are many varieties, and in Costa Rica six are being worked on. It is used to uncompact the soil, for animal and human nutrition and a biological control agent. As a conclusion, it can be used to improve the quality of the soils, protect water basins and nutrition for humans and animals.

Key words: Nitrogen Fixation, Family Diet, agrosilvopastoral, Food Security and Nutrition Systems, soil protection.

\section{Métodos y Materiales}

Se realizó una revisión bibliográfica que permitiera dar sustento teórico a este trabajo, se consultaron tanto fuentes físicas (bibliotecas) como virtuales (Web), que proporcionaran datos concretos sobre el Gandul, sus cualidades y sus rendimientos productivos.

En cuanto a la información recopilada del proyecto sobre sistemas agrosilvopastoriles que la Universidad Estatal a Distancia (UNED) ha implementado en San Carlos, se enfoca en los resultados obtenidos en el último año del proyecto. Las etapas se pueden dividir de la siguiente forma:

\section{Análisis Documental sobre la Situación de la Zona bajo Estudio}

Trabajo básicamente documental (revisión bibliográfica).

\section{Determinación del Uso Actual y Potencial de la Tierra en la Zona Bajo Estudio}

Trabajo cartográfico cuyo resultado fue la determinación de los ecosistemas de la cuenca. 


\section{Caracterización de la Población Meta}

Se realizó una caracterización de la población meta, con lo cual se sentará la información base necesaria para los procesos de extensión e investigación que se ejecutará durante el desarrollo del programa.

\section{Implementación de Sistemas Agroforestales y Proceso de Extensión}

Esta fase la constituye la implementación de los sistemas agrosilvopastoriles, para lo cual, se brindó un proceso de extensión a los productores, con el fin de capacitarlos en estos sistemas. Adicionalmente a este proceso de extensión se desarrollaron las siguientes acciones:

i. Determinación del uso actual y potencial de la tierra, realizando el mapeo correspondiente.

Esta fase del proyecto se identificaron los ecosistemas (tipos de cobertura forestal) naturales (busques, humedales, etc.) y artificiales (cultivos, plantaciones, etc.) con que cuenta la cuenca del río Peñas Blancas. Para estos efectos se empleo la metodología desarrollada por el proyecto ECOMAPAS del Instituto Nacional de Biodiversidad (INBio), que consistió de los siguientes pasos:

- Planificación y Cartografía

- Adquisición de las fotografías aéreas Carta 2003 de la zona.

- Georeferenciación, ortorectificación y fotointerpretación las fotografías aéreas Carta 2003.

- Elaboración del mapa de coberturas.

- Verificación de campo

- Verificación de la clasificación de la cobertura vegetal realizada desde la oficina.

- Descripción vegetal del ecosistema. Para lo cual se realizo un pequeño recorrido siguiendo una línea recta a lo largo de la cobertura forestal levantando una lista de las especies vegetales encontradas. Las especies no identificadas en el campo se colectaron e identificaron posteriormente en un herbario.

- Por cada ecosistema se realizaron al menos dos muestreos.

ii. Selección de las Especies a Reproducir en Vivero

Las especies a seleccionar debieron cumplir algunas de las siguientes cualidades:

- Ser fuente de alimento para la fauna silvestre de la zona.

- Poseer un sistema radicular que de sostén a terrenos con pendientes fuertes y degradados.

- Aportar al suelo beneficios como fijación de nitrógeno, mejora de la estructura (sistema radicular profundo), etc.

- Permitir su desarrollo con especies de uso agrícola bajo el esquema de sistemas agroforestales.

- Fuente de alimento forrajero.

- Producción de fuentes de alimento para el ser humano (semillas, hojas, raíces, etc.).

- Porte, estructura, floración y fructificación de la especie que mejore la belleza escénica del lugar.

iii. Selección de Árboles Semilleros

- Jerarquizar en la lista de mayor a menor interés las especies a ser reproducidas.

- Cada especie seleccionada fue ubicada en el mapa de cobertura.

- Se procedió a la ubicación en el campo de las especies antes mencionadas, determinando mediante coordenadas (GPS) la posición de los individuos con las mejores características fenotípicas y a los cuales se les denomino árboles semilleros.

- Se estableció un programa de monitoreo de los árboles semilleros a fin de determinar el momento en que fructifican y posteriormente cosechar los frutos.

- Una vez que los frutos estaban maduros se procedió a su recolección y posterior transporte al vivero. 
iv. Reproducción de Especies en Vivero

Esta fase se divide en los siguientes pasos:

- Preparación de las camas de germinación y los almacígales.

- Preparación de los sustratos para las camas de germinación y los almacígales.

- Desinfección de los frutos provenientes del campo.

- Secado de los frutos.

- Extracción de las semillas de los frutos.

- Siembra de las semillas en los almacígales y camas de germinación.

- Repique de las plántulas al sistema de reproducción (bolsas y platos jiffy).

v. Distribución del Material Reproducido en el Vivero

Una vez que las plántulas producidas en el vivero están listas para salir al campo para ser sembradas, se procede a realizar su distribución entre los habitantes de la localidad, esta distribución se hace de dos formas:

- A solicitud de un propietario que desea reforestar su finca.

- Se determina mediante los proyectos que se tienen entre el ICE y los propietarios de tierras, cuales son los idóneos para recibir el material y su posterior siembra en el campo.

vi. Ensayo en el Centro de Investigación, Transferencia Tecnológica y Educación para el Desarrollo (CITTED).

Con el fin de poder generar datos que permita dar información más acertada sobre la producción del Gandul según las condiciones locales, se realizó un ensayo de investigación en las instalaciones del CITTED. La metodología consiste en plantar varias parcelas con gandul bajo condiciones distintas, como suelo, sombra, humedad, etc. Este mismo diseño fue adoptado por varios productores de la zona a fin de experimentar ellos mismos con el Gandul, con la supervisión de funcionarios del ICE y de la UNED.
En total se experimentó con 12 variedades de Gandul.

Inicialmente en el CITTED se establecieron tres parcelas experimentales con gandul, sobre las cuales se llevaron registros de crecimiento y producción de semillas. Para el año 2013 se estableció una parcela por cada variedad de Gandul de las 6 que mejores resultados dieron en las fincas de los productores. Estas variedades fueron: Catie (negra), panamá (pintada), criolla (color crema), sabalitos (colores café y pintado) y enana. Se realizaron visitas periódicas al CITTED a fin de observar los resultados obtenidos en las parcelas, así como reuniones con funcionarios del ICE a fin de mantener un control sobre la actividad de producción y distribución de material a los productores de la zona.

vii. Talleres de Gestión

Con el fin de promover el proyecto e incentivar al finquero a producir con una nueva alternativa productiva amigable con el ambiente, fue que se desarrollaron un par de talleres con las comunidades locales (Jauuri y Sonafluca).

viii.Establecimiento de ensayos para investigación y producción.

Establecimiento, implementación y seguimiento de sistemas agroforestales en las fincas de los productores beneficiarios.

\section{Monitoreo de sistemas agrosilvopastoriles} implementados

Todas las actividades son desarrolladas de forma conjunta con los productores, en las cuales participará todo el equipo de trabajo y los estudiantes de grado y posgrado que realicen sus trabajos finales dentro del proyecto. Se pretende que el productor sea un coinvestigador y participe de manera activa en la administración de su finca a la vez que hace más sostenible la misma tanto económica como ambientalmente. 
Establecimiento del banco de germoplasma en el CITTED

Se establecieron seis parcelas dentro de las instalaciones del CITTED con seis variedades de Gandul. Estas variedades fueron obtenidas de las fincas de los productores que son parte del Proyecto sobre Sistemas Agrosilvopastoriles que la UNED desarrolla en colaboración con el ICE. De las 12 variedades con las cuales se implemento el proyecto se seleccionaron seis, las cuales durante los últimos 3 años mostraron una excelente adaptación a las características del lugar y cuyos rendimientos en la producción de frutos fueron bastante exitosos.

La distribución espacial de las parcelas dentro de la propiedad del CITTED dependiendo básicamente de la disponibilidad de espacio en el lugar, fundamentalmente se le dio prioridad a los sitios que tuvieran la mayor disponibilidad de entrada de luz y que a su vez estuvieran menos presencia de árboles o arbustos. Para la generación de información se diseño un protocolo con el cual se capturaron los datos que se consideraron de importancia, entre ellos: fecha de la actividad (siembra, cosecha, floración, etc.), mortalidad, germinación, crecimiento diamétrico, crecimiento en altura, floración, fructificación, cosecha, plagas y enfermedades, peso de la cosecha (vainas, semillas).

\section{Resultados}

\section{Descripción del Gandul}

Según Morton et al. (1982) y van der Maesen (1990) citados por Soto Vélez (1997), el gandul es un arbusto que alcanza una altura de hasta 5 metros y su tallo tiene un diámetro aproximado de 1 a 4 centímetros. La raíz principal de este arbusto es capaz de alcanzar una profundidad de hasta $3 \mathrm{~m}$. La raíz principal produce raíces secundarias que pueden producir nódulos capaces de albergar bacterias nitrificantes.

Omanga y Summerfield (1994) citado por Soto Vélez (1997) indica que el sistema radicular vigoroso de gandul le permite a la planta subsistir bajo condiciones adversas, donde la disponibilidad de agua y nutrientes no sean adecuadas para el desarrollo de otras especies. Por otro lado, existen muchas variedades y tipos que difieren entre sí por su precocidad, resistencia a enfermedades y sequía; se conocen más de 30 variedades (Ávila C, Cedillo E; Cervantes C; 2008).

Es característico de esta especie sus flores amarillas con rayas cafés o pardas. Sus frutos son de color moteado con pardo y pubescentes, con el ápice curvado; sus semillas son de color pardas y moteadas de blanco (Hammel, Grayum, Herrera, Zamora; 2004-2007). Las vainas (fruto) son indehiscentes y cuando están inmaduras pueden tener un color verde con manchas marrón-púrpura y al madurar se tornan de un verde amarillento (van der Maesen, 1990). Usualmente albergan de 2 a 9 semillas que tienden a ser redondos con un diámetro de 4 a 8 $\mathrm{mm}$. Cuando la semilla está inmadura es verde y cuando éste madura tiende a ser amarillo, blanco o gris aunque hay variedades donde los granos son de colores púrpura, negro y rosa (Morton et al., 1982).

\section{Distribución del Gandul}

El gandul es originario de la India y Cercano Oriente (Avila C, Cedillo E; Cervantes C; 2008). Es común en Costa Rica del Bosque húmedo, es una especie nativa de India, ampliamente cultivada por sus semillas comestibles y a menudo naturalizadas (Hammel, Grayum, Herrera, Zamora; 2004-2007). Esta especie se encuentra en África, desde Zambia hasta las costas de Angola; en Asia se cultiva en China, Vietnam, Laos, Camboya, India y Malasia; crece en las montañas de la parte central de Java y en América en las partes cálidas. Se distribuye también en Birmania, República Dominicana, Uganda y Malawi. En México en los municipios de Matías Romero, Oax. y Santa María Chimalapa, Oax (Ávila C, Cedillo E; Cervantes C; 2008).

Morton et al. (1982) citado por Soto Vélez (1997) indica que a principios de siglo XVIII el cultivo del gandul se encuentra bien establecido en China, Indochina e India Oriental de donde luego se dispersa hacia las Islas del 
Pacífico. Posteriormente es transportado a través de la ruta de esclavos de África hacia las Bermudas, Indias Occidentales, Guineas y Brasil. Según van der Maesen (1990) citado por Soto Vélez (1997), el gandul probablemente fue domesticado de la especie Cajanus cajanifolius (Haines). Actualmente la planta se encuentra distribuida y naturalizada a través de todas las regiones tropicales.

\section{Usos del Gandul}

El gandul se cultiva hace unos 3000 años, posee una su raíz penetrante bastante útil para descompactar los suelos. Es empleada en la alimentación de humanos y animales (como forraje), posee un alto valor nutritivo cuyo contenido de proteína varía entre el 18 y $25 \%$, algunas variedades alcanzan hasta un $32 \%$. Puede fijar entre 41-280 Kg. de N/ha/año, sus ramas y vástagos son utilizados como combustible (Vivas \& Morales, 2004). En Paraná (Brasil), esta especie es una de las que posee un mayor potencial para la alimentación de cerdos y aves en propiedades de autoconsumo. En la India, Uganda, Bahamas, Panamá y Brasil, el gandul es cultivado con el fin de utilizar sus granos secos para consumo humano debido a su alto contenido de proteína que oscila entre 18 y $25 \%$ (Morel \& Piccolo, 2002). Las semillas también son fuente de alimento (proteína) para las aves de corral. Se ha incluido hasta un $30 \%$ en las raciones de alimento para los pollos, con igual ganancia de peso que en el caso de los pollos mantenidos con una mezcla isonitrogenada de harina de soja y maíz (ONU, 2008).

Algunas investigaciones realizadas en Santa Catarina (Brasil) determinaron que el gandul produce hasta 60 toneladas de forraje verde, conteniendo 15 toneladas de materia seca con $17 \%$ de proteína bruta. Por lo cual, esta especie es recomendada como banco de proteína para la suplementación animal (Morel \& Piccolo, 2002). Pero el gandul también es empleado en el consumo humano por su alto contenido de proteína, promediando un $7 \%$ cuando la semilla se encuentra inmadura y de 18 a $32 \%$ cuando está madura. Tiene un buen balance entre todos los aminoácidos, con la excepción de metionina, cisteína y triptófano. Cuenta con un alto contenido de vitaminas A y $\mathrm{C}$, también está presente el elemento fósforo en concentraciones de 200 mg.100 g-1 de semilla seca (Soto Vélez A, 1997).

Sus frutos, semillas y hojas constituyen un excelente forraje para el ganado bovino. También es empleado para heno y ensilaje (a menudo, con melaza); para este objeto se emplean las variedades de grano pequeño. Si se siega para heno cuando los frutos (vainas, legumbres) están bien desarrolladas, las plantas pueden cortarse cada vez con una mayor altura (ONU, 2008). Debido a la toxicidad de sus raíces, esta especie es plantada como seto alrededor de los sembrados de yuca, para protegerlos contra los topos; también es cultivada alrededor de las casas con el propósito de protegerlas contra las termitas (ONU, 2008). Es también empleado como agente de control biológico de malezas muy agresivas (v.gr. Saccharum spontaneum), pese a ser una especie de alto consumo mundial, es muy poco estudiada a nivel biotecnológico (Montero, Macías y Wong; 2006).

Según Soto Vélez A (1997) el gandul es utilizado en Hawaii, Inglaterra y otros países como alimento para ganado. En Hawaii es utilizado como forraje sustituyendo la alfalfa. En estos países se prefiere utilizar el gandul como forraje por su contenido en proteína cruda, pero el grano no es utilizado comúnmente porque éste es deficiente en ciertos aminoácidos y tendrían que ser añadido aditivos costosos. En regiones de India y África el gandul es utilizado como combustible. También se ha estudiado la utilidad del gandul como abono verde, rompe vientos y cobertora por ser hospedera de ciertas plagas como el gusano de seda. Si el gandul es inoculado con la bacteria Rhizobium puede formar nódulos en las raíces siendo capaz de incorporar al suelo entre 41 a $280 \mathrm{~kg} / \mathrm{ha}$ de nitrógeno.

\section{Requerimientos y Composición del Gandul}

Se adapta a diferentes condiciones de suelo y clima, además es poco exigente en fertilización. Se desarrolla en un amplio rango de altitud, el cual oscila de 0 a 2000 msnm, la altura 
óptima va de 0 a $800 \mathrm{msnm}$; crece en un rango de precipitación que va de moderadas a altas (de 700 a $1500 \mathrm{~mm}$ y de 530 a $4020 \mathrm{~mm}$ con óptima de 700-2000 $\mathrm{mm}$ ); prospera en temperaturas de 16-35 ${ }^{\circ} \mathrm{C}$, con una óptima de 18 a $28{ }^{\circ} \mathrm{C}$; tolera la sequía y crece en suelos pobres y con poco contenido de fósforo, con textura franca-arenosa a franca-arcillosa, de fertilidad baja o moderada, con $\mathrm{pH}$ moderadamente ácido y neutro (5-7.5), desarrollándose mejor en $\mathrm{pH}$ de 5.0-7.0 (Ávila C, Cedillo E; Cervantes C; 2008).

Para garantizar un alto porcentaje de germinación es necesario realizar tratamientos a las semillas, entre ellos se mencionan la escarificación mecánica, la química, tratamientos térmicos y el almacenamiento de las semillas (Pietrosemoli, 1997).

La semilla de gandul posee tripsina que es un compuesto químico que puede ocasionar la muerte de animales domésticos que se alimenten de ellos; sin embargo cocinando el gandul se mejoraron los rendimientos de los animales al destruirse las sustancias inhibidoras de la tripsina.

El gandul es empleado en el consumo humano por su alto contenido de proteína, promediando un $7 \%$ cuando la semilla se encuentra inmadura y de 18 a $32 \%$ cuando está madura. Tiene un buen balance entre todos los aminoácidos, con la excepción de metionina, cisteína y triptófano. Cuenta con un alto contenido de vitaminas A y C, también está presente el elemento fósforo en concentraciones de $200 \mathrm{mg} .100 \mathrm{~g}$-1 de semilla seca (Soto Vélez A, 1997).

\section{Rendimientos y Producción del Gandul}

Se han obtenido datos de producción que van de 20 a 40 t/ha/año de forraje fresco y de 9 a 12 t/ha/año de materia seca. Un ejemplo es Puerto Rico, en donde se ha producido materia seca en un $24.4 \%$ de la cual el $21.4 \%$ es proteína cruda y $30.8 \%$ fibra cruda; en Hawaii, la planta en fase lechosa contiene $49.7 \%$ de materia seca, del cual el18.9 \% es proteína cruda y el $29.7 \%$ fibra cruda; finalmente en Trinidad y Tobado las cáscaras de las legumbres del gandul contienen $93 \%$ de materia seca, de este porcentaje el 6.7 $\%$ es proteína cruda y el $38 \%$ fibra cruda. El rendimiento de grano va de un 0.8 a 1.4 t/ha. También se han obtenido datos importantes en la fijación de nitrógenos que el gandul proporciona al suelo, estos valores van de 41 a $280 \mathrm{~kg}$ de N/ ha/año (Ávila C, Cedillo E; Cervantes C; 2008).

Según Avila V. (inédito) el gandul enano produce 5.74 toneladas por hectárea de materia orgánica con los siguientes aportes de nutrientes: nitrógeno $2.77 \%$, fósforo $0.35 \%$, calcio $1.05 \%$, magnesio $0.25 \%$, y potasio $2.21 \%$ en materia orgánica. El equivalente de estos valores en abono químico seria: nitrógeno 9.5 sacos de Nutrán, fósforo 2 sacos de 18-46-0, y potasio 9.8 sacos de 15-3-31. Además se reporta que a un distanciamiento de 1 x 0.5 metros el gandul produce 10.61 toneladas de materia orgánica por hectárea; con una producción de nutrientes de la siguiente manera: nitrógeno $179.6 \mathrm{Kg} / \mathrm{ha}$, fósforo $25.5 \mathrm{Kg} / \mathrm{ha}$, calcio $90.4 \mathrm{Kg} / \mathrm{ha}$, Magnesio 23.4 $\mathrm{Kg} / \mathrm{ha} \mathrm{y} 72.3 \mathrm{Kg} / \mathrm{ha}$.

Según Soto Vélez (1997) la producción de gandul en Puerto Rico para el periodo 20052006 fue de 4841 quintales en granos verdes con un valor de $\$ 1539000$. En Puerto Rico el consumo per-cápita promedio del gandul es de 2.23 libras, de éstos el $75 \%$ es consumido como gandul procesado. Localmente se produce el $4.3 \%$ del gandul consumido, el restante es importado de países como: Ecuador, Perú, Santo Domingo y recientemente Brasil. Localmente la producción de gandul enlatado, tiene un costo promedio de $\$ 18$ por una caja de 24 latas, mientras que el grano importado tiene un valor de \$12 la caja.

\section{Plagas y Enfermedades del Gandul}

Las plagas y enfermedades que más frecuentemente atacan al gandul son: Fusarium odum Butl, Helionthis armigera Hubn, Exelastis atomosa W., Agromyza obtusa M., Elasmopalpus rubedinellus Zell, Anoylostomia stercurea Zell, y Helionthis virescens F., Spodoptera spp., Estigmene acrea, Trichoplusia ni, Diabrotica spp., Epilachna varivestis, Hemisia tabaci, Aphis craccivora, Empoasca kraemeri, Cory thucha gossypii, Coccus sp., Elasmopalpus lignosellus, Helicoverpa zea, Maruca testulalis, Acanthoscelides obtectus y Nezara 
viridula; también llega a sufrir enfermedades causadas por Alternaria fasciculata, Botrytis sp., Colletotrichum dematium, Phoma exigua, Physalospora cajanae, Phythophthora parasitica, Sclerotinia sclerotiorum, Uromyces dolicholi, Xanthomonas cajani, Fusarium udum y Rhizoctonia solani; en nemátodos específicamente estan: Helicotylenchus microcephalus, H. cavenessi, H. pseudorobustus, Hoplolaimus seinhortii, Meloidogyne javanica, M. arenaria, M. incognita, Pratylenchus sudanensis y Rotylenchus reniformes (Ávila C., Cedillo E.; Cervantes C.; 2008).

Otras plagas y enfermedades detectadas en Panamá son: Alternateria alternata (Fr.) Keissl. (Mancha Foliar); Cladosporium oxysporum Berk. \& M.A. Curtis. (Tizon Foliar); Colletotrichum cajani Rangel. (Antracnosis); Fusarium equiseti (Corda) Sacc. (Damping-off); Fusarium sp. (Marchitez); Macrophomina phaseolina (Tassi) Goidanich. (Pudricion Seca del Tallo); Myrothecium roridum Tode:Fr. (Mancha Foliar); Mycovellosiella cajani (Henn) Rangel ex Trotter (Mancha Foliar); Periconia byssoides Pers. (Mancha Foliar); Phaeoisariopsis griseola (Sacc.) Ferraris. (Mancha Foliar); Phyllosticta cajani Rangel. (Mancha Foliar); Pythium aphanidermatum (Edson) Fitzpatrick (Dampingoff); Rhizoctonia solani Kühn. (Pudrición de Raíces); Sclerotium rolfsii Sacc. (Pudrición del Tallo); Uredo cajani Sydow. (Roya); Micoplasma-Roseta; Micoplasma (Escoba de bruja) (Esquivel R., 2007). Contarinia sp. (mosca de follaje) puede destruir el $90 \%$ de las flores y Melaganagromyza obtusa (mosca de la vaina del gandul) y Contarinia sp. (mosca del follaje) que puede destruir el $90 \%$ de las flores (Soto Velez A, 1997)".

Se presenta una muy breve reseña de los resultados obtenidos del proyecto de Sistemas agrosilvopastoriles en la Cuenca del Río Peñas Blancas, en sus inicios

Se identificaron los ecosistemas naturales (bosques, humedales, etc.) y artificiales (cultivos, plantaciones, etc.) con que cuenta la cuenca del río Peñas Blancas. Obteniéndose los siguientes resultados:

- Coberturas naturales. El análisis permitió identificar dos tipos de bosques, los densos y los ralos, estos a su vez abarcan tres pisos altitudinales, basal, premontano y montano bajo (Herrera y Gómez, 1993), generando los siguientes tipos de cobertura: Bosque denso montano bajo, Bosque denso premontano, Bosque denso basal.

- Coberturas seminaturales. Se caracterizan por la evidente intervención humana. Esta compuesta por bosques secundarios incipientes, bosque secundarios en etapas intermedias y bosque maduros muy intervenidos por la actividad maderera. Se les ha clasificado en: matorrales densos, matorrales ralos o matorrales densos arbolados, esto según sea la dominancia de árboles o arbustos.

- Coberturas culturales. Son sitios de actividades productivas o de subsistencia para el ser humano (pastizales, cultivos agrícolas, plantaciones forestales, plantaciones de frutales, etc.). Se dividen en herbazales (arbolados, arbustivos, densos y ralos). Las otras actividades se identificaron con el nombre específico del cultivo (yuca, plátano, arroz, plantación de melina, plantación de mamón chino, etc.).

La cuenca del río Peñas Blancas posee un área total de $400 \mathrm{Km}^{2}$ con un rango de elevación entre 25 y 1900 m, con una precipitación que varía de 1600 - $6500 \mathrm{~mm}$ anuales compuesta por 25 comunidades que conforman una población de 13500 habitantes. Esta cuenca posee características agropecuarias bien definidas que permite hacer una clara diferenciación en tres sectores, alto, medio y bajo. A su vez estos sectores revelan una variedad de condiciones de suelo, clima, relieve y cultura agropecuaria.

Se reprodujeron las siguientes especies nativas para ser distribuidas entre los pobladores y recuperar parte de las áreas más degradadas: 
CUADRO 2

\section{Especies reproducidas en vivero}

\begin{tabular}{lcc}
\multicolumn{1}{c}{ Especie } & $\begin{array}{c}\text { Cantidad } \\
\text { producida }\end{array}$ & $\begin{array}{c}\text { Germinación } \\
(\%)\end{array}$ \\
\hline Protium sp. & 10 & 30 \\
Inga sp. & 50 & 40 \\
Posoqueria latifolia & 50 & 60 \\
Ocotea sp. & 200 & 80 \\
Iriartea deltoidea & 300 & 80 \\
Caesalpinia sp. & 500 & 70 \\
Pouteria sp. & 500 & 80 \\
Dendropanax arboreus & 1000 & 78 \\
Swartzia nicaraguensis & 1000 & 80 \\
Hymenaea courbaril & 1500 & 80 \\
Cajanus cajan & 3500 & 95 \\
Ardisia revoluta & 5000 & 90 \\
Cordia cymosa & 6000 & 87 \\
Lonchocarpus sp. & 10000 & 85 \\
Zygia longifolia & 40000 & 90 \\
\hline
\end{tabular}

Dentro de los resultados obtenidos en el componente de extensión del proyecto, a través de los diversos talleres que se impartieron con algunos productores interesados en ser parte del proyecto, fue que un pequeño grupo de ellos se organizaran y conformaran formalmente una asociación, la cual denominaron "Asociación de Productores de Gandul y tubérculos".

\section{Discusión}

Son notables los beneficios que brinda el cultivo del Gandul en su gran gama de variedades, en términos generales, vemos que sus aportes al ambiente y a la Sociedad en cuanto a los sistemas productivos son de gran valor, entre los que podemos mencionar: brinda una cobertura forestal al suelo evitando la erosión por impacto directo de la lluvia, su sistema radicular mejora la estructura del suelo favoreciendo la infiltración de las aguas superficiales y evitando con ello la erosión por escorrentía, al mejorar la infiltración también nutre a las aguas subterráneas, al fijar nitrógeno al suelo incrementa las condiciones de este, lo cual se traduce en mejores rendimientos en la producción de otros cultivos, brinda un aporte importante de proteínas a la dieta cotidiana, proporciona forraje para el ganado, alimento para animales de corral, abono verde, remuneración económica al núcleo familiar por la venta de sus productos, etc.

En lo que respecta a coberturas naturales la Cuenca del Río Peñas Blancas cuenta aún con un importante recurso forestal ya que un $56.65 \%$ de su superficie está cubierta por bosques, básicamente densos y ralos.

De los muestreos y de la caracterización de la cobertura forestal de la Cuenca se selecciono un total de 69 especies arbóreas potenciales para ser reproducidas y distribuidas entre los productores locales a través de una campaña de reforestación a fin de mejorar las características ambientales de la zona. De estas especies se lograron reproducir en vivero 15 especies, para un total de 69610 individuos, los cuales se distribuyeron exitosamente entre los pobladores locales para la reforestación en sus fincas, principalmente alrededor de fuentes de agua.

En lo pertinente a la viverización y siembra del Gandul bajo sistema agrosilvopastoril se contó con el apoyo de 12 fincas de productores de la zona, a quienes se les distribuyó las plántulas listas para ser plantadas. Periódicamente se realizaron visitas de seguimiento a las fincas, sobre todo en las épocas de cosechas. Tanto la adaptación del Gandul como las cosechas fueron un éxito, pese a que se notaron diferencias marcadas según la variedad. En lo que respecta a la comercialización del producto se han encontrado problemas a nivel nacional para colocar el producto, por ser un producto nuevo para la dieta familiar.

Con respecto al desarrollo de productos innovadores a base de gandul, como se ve en los resultados, se ha logrado introducir el producto a panelistas del área metropolitana de Costa Rica mediante paneles sensoriales, grupos focales y ferias de degustación y venta. Los productos que se han desarrollado han sido en general aceptados por los consumidores y se ha visto una ligera tendencia a ser elegidos por encima de la competencia. 


\section{Agradecimientos}

Se extiende un especial agradecimiento a la Ing. For. Mery Ocampo Araya por el enorme apoyo que le dio a este proyecto, el cual sin su ayuda no hubiese podido concretarse, se extiende además este agradecimiento a los ingenieros Herberth Villalobos Soto y Jorge Escribano Montoya, funcionarios del ICE. Además, se agradece a los productores de la Asociación de productores de gandul, raíces y tubérculos de San Carlos por su dedicación y apertura a forjar poco a poco este proyecto. También se le agradece a los panelistas y participantes de todas las pruebas sensoriales y de mercadeo (grupos focales) que se realizaron.

\section{Referencias Bilbiográficas}

Ávila C, Cedillo E; Cervantes C. 2008. Base de Información sobre especies con Potencial de Abonos Verdes y Cultivos de Cobertura (En Línea). Red de Grupos de Agricultura de Cobertura, Mexico. Consultado 30 Oct. 2008. Recuperado de: Http://Cajanus. EML/Abonos\%20www.virtual.chapingo.mx.pdf/ C58EA28C-18C0-4a97-9AF2-36E93DDAFB3/ Abonos\%20www.virtual.chapingo.mx.pdf?attach $=1$

Avila Vega J (inédito). Uso de abonos verdes, Experiencias en la Región Huetar Norte. Inedito, Costa Rica. 8 páginas.

Herrera, W. Gómez y LD. 1993. 1993. Mapa de Unidades Bióticas de Costa Rica. Mapa de Unidades Bióticas de Costa Rica. Escala 1:685.000. Escala 1:685.000. US Fish and Wildlife Service - TNC - INCAFO - CBCCR - INBio - Fundación Gómez-Dueñas.
EE.UU. Fish and Wildlife Service - CNC - INCAFO - CBCCR - INBio - Fundación Gómez-Dueñas. San José. San José.

Hammel, B.E.; Grayum, M.H.; Herrera, C.; Zamora, N. (eds.). Manual de Plantas de Costa Rica. 2004-2007. Vols. I-IV. Monogr. Syst. Bot. Missouri Bot. Gard.

Morel F. y Piccolo G. 2002. Evaluación del "Guandú" para recuperar la fertilidad del suelo (en línea). Buenos Aires, Argentina. Consultado 17 oct. 2008. Recuperado de: http://www.inta.gob.ar/cerroazul/investiga/suelos_anuales/guandu.htm

Organización de Las Naciones Unidas para la Agricultura y la Alimentación, 2008. Cajanus cajan (en línea). Consultado 17 oct. 2008. Recuperado de: http://www. oei.org.co/oeivirt/edumat.htmhttp://www.fao.org/AG/ aGa/agap/FRG/AFRIS/Es/Data/205.HTM

Pietrosemoli S. 1997. Efecto del Almacenamiento y la Escarificación sobre ILa Germinación de la Semilla de Quinchoncho Cajanus Cajan (L.) Millsp (en línea). Facultad de Agronomía, Venezuela. Consultado 30 oct. 2008. Disponible en http://www.alpa.org.ve/PDF/ Arch\%2005\%20Suplemento/PF09.pdf

Soto Vélez A, 1997. Sincronizacion de Florecida en Cajanus cajan (L.) Millsp. Mediante el uso de Biorreguladores. Maestro En Ciencias. Mayagüez, Puerto Rico. Universidad de Puerto Rico. 84 págs.

Vivas Q. N. y Morales V. S. 2004. Evaluación Agronómica y producción De Grano De Diez Accesiones De Guandul (Cajanus Cajan) en la Meseta de Popayán - Cauca (EnLínea). Popayán-Colombia, Universidad del Cauca. Consultado 17 Oct. 2008. Recuperado de: Http://Www.Unicauca.Edu.Co/Biotecnologia/ Ediciones/Vol3/Art35.Pdf 
